\title{
A Brief Tour to Ethical Guidelines
}

\author{
Prof. Ritu Prasad Gartoulla, Ph.D. \\ Chairman \\ Institutional Review Board, Research Department \\ Institute of Medicine, TU, Kathmandu, Nepal
}

Many students and teachers under the campuses and hospitals of the Institute of Medicine, Tribhuvan University and other colleges affiliated to different universities are unaware about the Ethical considerations of their research and publications. They feel burden on the issues of Institutional Review Board/committees as to they should submit and meet all the requirements adopted by the concern IRB/Cs. But, without ethical considerations, no any researches are recognized by any institutions. Ethical consideration means recognizing the Human Rights in Health sector where Human are the subject for study.

Nepal Health Research Council, a governing National autonomous body on health related researches has contributed significantly promoting ERB and IRC/IRBs in the country. NHRC has a very comprehensive and concise publication on "National Ethical Guidelines For Health Research in Nepal and Standard Operating Procedures" (Published in January 2011). It is like a Manual for all researchers and organizations.

This brief tour to ethical guidelines only provides basic general information to highlight the important points which might help researchers to understand in brief about Ethics. These points have been cited (without permission of concern persons) like the tips of Forum for Ethical Review Committees in Asia and the Western Pacific (FERCAP) Workshop in July 2011, Bangkok, Thailand and points presented by different organizations at the Consultative Meeting on Research Review Process organized by NHRC and Mary knoll Fathers and Brothers Project (9-10 September 2011) and from other references.

Thus, I am happy to provide a basic information regarding Ethics.

\section{History}

\section{The Nuremberg Code}

Principles resulting from the Nuremberg Trials

- $\quad 23$ leading German physicians and administrators tried for conspiracy, war crimes, crimes against humanity, membership in criminal organization

- 16 sentenced to either death by hanging or imprisonment; 7 were acquitted

The Nuremberg Code explains major 10 points for ethics

1. Voluntary consent of the human subject

2. Experiment results should be for the good of society
3. Experiment on humans should be based on animal experimentation

4. Experiment should avoid unnecessary physical and mental suffering/injury

5. Experiment should not be done if it will cause death/ disability

6. Risk must not exceed humanitarian importance of research

7. Proper preparations \& adequate facilities

8. Experiment done only by qualified persons.

9. Subjects should be free to end participation

10. Scientist must be prepared to terminate the experiment at any stage, if needed

\section{Nuremberg Code: Impact}

1. UNHCR: general human right not to be a subject of experimentation "against one's will"(1948) or "without one's free consent" (1952 and 1958)

2. Declaration of Geneva: condemned the Nazi experiments

3. Geneva Conventions I,II,III,IV: protections against unwanted biological or medical experimentation

\section{Declaration of Helsinki}

- Morally binding obligation for physicians - overrides national laws/legislations, thus a higher standard

- Original rationale - no international statement on human experimentation;

- $\quad$ limited laws

- $\quad$ Expanded the 10 Nuremberg Principles

Basic text for international guidelines such as CIOMS, WHO Guidelines for (Good Clinical Practice) GCP for trials, ICH Tripartite Guideline for GCP

- Council of Europe

- $\quad$ Adopted by several National guidelines worldwide

- $\quad$ Amended 1975, 1983, 1989, 1996, 2000, 2008

\section{Provisions}

1. Scope (Art 1): statement of principles for medical research involving: Human subjects, identifiable human material, Identifiable human data

2. Overall duty of the physician (Art 2,3,4,10,11)

3. Purpose of research and the protection of human subjects (Art 5,6,7) 
4. Risks and burden (Art 8) and ethical responsibility (Art 9)

5. Risks \& benefits assessment (Art 18, 20, 21)

6. Scientific standards and design (Art 12, 14, 16)

7. Welfare of animals (Art 12) and protection of the environment (Art 13)

8. Oversight by a research ethics committee (Art 15,19

9. Research on vulnerable subjects (Art 9,17)

10. Respect for persons:

- Autonomy and elements of consent

- Voluntariness (Art 22)

- $\quad$ Privacy and confidentiality (Art 23)

- Disclosure of information and documentation of consent (Art 24)

- $\quad$ Duress (Art 26)

- Incompetence to consent (Art 27, 28, 29)

- Use of identifiable human material/data (Art 25)

11. Publications (Art 30)

12. Additional Principles

- $\quad$ Research with medical care (Art 31)

- Use of placebo (Art 32)

- $\quad$ End of study entitlements (Art 30)

- Treatment vs Research (Art 34)

- Other requirements if no proven interventions exist (Art 35)

Council for International Organizations of Medical Sciences (CIOMS)

1. CIOMS is an NGO established by WHO and UNESCO in 1949

2. Promotion of international research activities in the biomedical sciences

3. Programmes: Bioethics, Health Policy, Drug Development, and International Nomenclature of Diseases

\section{CIOMS Guidelines}

1. Formally known as the International Ethical Guidelines for Biomedical Research Involving Human Subjects

2. Created 1993 by the Council for International Organizations of Medical Sciences (CIOMS); updated in 2002

3. 21 guidelines (15 in the original report) address different ethical issues

CIOMS: Guidelines for Epidemiological Research

1. International Guidelines for Epidemiological Studies

2. Created 1991; published updated version in 2009

3. 24 guidelines (originally 53 provisions, thematically grouped)

\section{CIOMS Guidelines explains for}

1. Scientific validity

2. Ethical review committee (local review)
3. Individual informed consent

4. Undue inducement

5. Benefits/risks to participation

6. Populations/communities with limited resources

7. Equitable distribution of burdens and benefits

8. Vulnerable persons

- $\quad$ Children

- Mental/behavioral incapacitated to consent

- Women

- Pregnant women

9. Confidentiality

10. Right of injured to treatment \& compensation

11. Strengthening capacity for review

12. Obligations of sponsors

\section{CIOMS Guidelines: Impact}

"To indicate how the ethical principles that should guide the conduct of biomedical research involving human subjects, as set forth in the Declaration of Helsinki, could be effectively applied, particularly in developing countries, given their socioeconomic circumstances, laws and regulations, and executive and administrative arrangements".

\section{Some Challenges}

Guidelines are based on Western values/principles and are sometimes problematic when applied to non-Western countries. Example: Individual informed consent \& autonomy

1. In some communities, women must ask for permission from their husbands

2. In some communities, community permission is required

3. The universal nature of guidelines is challenged by specific requirements in local context and in specific researches

4. However, standards in the form of guidelines contribute to having an ethical framework in the conduct of research (especially across countries)

The Role of Ethics Committees in Health Research

\section{Health Research as a Social Activity}

1. Research involves human beings

- Sponsors/researchers vis-à-vis research subjects

- Industry vis-à-vis regulatory officials

- Research stakeholders vis-à-vis general public

2. Ethics - principles of conduct governing human relationships

3. Need for guidelines/regulations for mutual benefit

4. Need for multi-stakeholder perspective

5. Principle of check and balance

\section{Ethical Concerns}

- $\quad$ The booming clinical trial industry is raising concerns because of: 
- A lack of regulation of private trials

- And the uneven application of requirements for informed consent

- And proper ethics review.'

- $\quad$ Specific concern about lack of independence of ethics committees

\section{Therapy vs. Research}

- $\quad$ Therapy - objective of clinical practice

- Best therapy for patient

- Individualized

- Objective - use of most effective intervention

- $\quad$ Research - generalizable knowledge

- Standardized intervention

- Randomized design

- Experimentation

\section{Ethical Guidance for IRBs/IECs}

- International Guidelines

- Helsinki, Belmont, CIOMS, etc.

- National Laws and Guidelines

- $\quad$ Standard Operating Procedures

- WHO Operational Guidelines for Ethics Committees That Review Biomedical Research

- ICH-GCP

- $\quad$ Requirements for international assurances

- Federal wide Assurance (FWA), etc.

Declaration of Helsinki 2008 defines role of REC/ IRB

1. The research protocol must be submitted for consideration, comment, guidance and approval to a research ethics committee before the study begins.

2. This committee must be independent of the researcher, the sponsor and any other undue influence.

3. It must take into consideration the laws and regulations of the country or countries in which the research is to be performed as well as applicable international norms and standards but these must not be allowed to reduce or eliminate any of the protections for research subjects set forth in this Declaration

4. The committee must have the right to monitor ongoing studies.

5. The researcher must provide monitoring information to the committee, especially information about any serious adverse events.

6. No change to the protocol may be made without consideration and approval by the committee.

\section{Good Clinical Practice (GCP)}

An international ethical and scientific quality standard for designing, conducting, recording, and reporting trials those involve the participation of human subjects and for public assurance that the rights, safety, and well-being of trial subjects are protected.
WHO Operational Guidelines For Ethics Committees That Review Biomedical Research 2000

To contribute to the development of quality and consistency in the ethical review of biomedical Research Defines:

1. Membership requirements,

2. Training of members

3. Operating ERC guidelines

4. Meeting requirements

5. Knowledge of ethical issues

6. Independence from influences

\section{Composition of Ethics Committees}

1. At least 5 members

2. At least 1 member from non-scientific area

3. At least 1 member independent of the institution/trial site

4. Diversity of background for complete and adequate review

5. Representation of men and women.

6. Representatives from the old and young

\section{Selection of IRB/IEC Members}

1. Members selected based on their interest,

2. Ethical and/or scientific knowledge and expertise, as well as on their commitment

3. Disclosure of conflict of interest in a proposal under consideration.

4. Signing a confidentiality agreement at the start of their term

5. Appointment for a period of 3 years and renewable for up to two consecutive terms.

6. Rotation enforced to ensure continuity

\section{Independent Consultants}

1. Provide assessment or advice on specific protocols

2. Appointed by the Chairperson of the IEC/IRB.

3. Relevant professional qualifications required

4. Willing to disclose and publicize full name, profession, and affiliation, financial accountability

5. Willing to sign Confidentiality / Conflict of Interest Agreements regarding meeting deliberations, applications, information on research participants, and related matters.

\section{Training of Members}

IEC/IRB members should maintain Competence updating of their knowledge of:

1. Good Clinical Practice (GCP)

2. Declaration of Helsinki

3. Ethical Issues

4. Relevant laws

5. Developments in relevant science, technical and environmental, health and safety aspects 
6. Relevant requirements of health, safety and environmental laws and regulations and related documents

7. Audit procedures

8. IRB/IEC Responsibilities

\section{IRB/IEC Responsibilities}

1. Safeguard the rights, safety, and well-being of all trial subjects

2. Review research documents

3. Examine qualifications of investigators and staff

4. Review the PROTOCOL (technical review)

5. Evaluate study sites

6. Review study participation

7. Analyze risks (physical, psychological, social), Assess benefits, Involvement of vulnerable participants

8. Review Decisions (Approval/favorable, Modification required, Disapproval/negative opinion, Termination/ suspension of prior approval

9. Notify the investigator to report

10. Help monitor implementation

11. Retain all relevant records for at least 3 years after completion of the trial and Make them available upon request from the regulatory authorities

12. Protect rights, safety and well-being of the subjects

13. Transparency and objectivity, Accountability and responsibility,

\section{Ethics Committees need}

Ethics Committees need to ensure the ability to arrive at decisions that reflect the a full appreciation of the scientific and ethical concerns of the protocol through the use of High standards of ethical review that result from combined knowledge, practice and experience.

\section{IRB at IOM}

Based on the above statements and requirements, IOM has established IRB for:

1. Adapting International Ethical Laws, Regulations, Chapters, Declarations \& Guidelines along with Nepalese laws and regulations

2. Coordinating with different IRBs in the countries including NHRC and abroad

3. Analyzing in-house proposals and Proposals of PG students of Health Sciences

4. Ensuring jurisdictions of IRB and acting based on the need

5. Other as per the need and decision of the board meetings

\section{Problems encountered}

1. Lack of training of the board members on IRB issues

2. Lack of knowledge on Ethical issues of the board members

3. Absenteeism in the monthly meeting of the board members

\section{Lack of experts/reviewers}

5. Lack of trained administrative staffs and poor filing and recording system

6. Breaking confidentialities

7. External influences

8. Lack of incentives to the Board members

9. Lack of infrastructures

10. Lacking office equipments

11. Slow administrative process and long process in the administration

12. General lacking

IRB Members at IOM (2011 to for 3 years)

The Ex Chairman Prof. Dr. Puspa Raj Sharma and IRB team have contributed a lot to establish system in IRB/IOM

\section{The Present Team has composed of:}

1. Prof. Dr. Ritu Prasad Gartoulla, Ph.D. Medical Anthropologist

2. Prof. Dr. Pradip Vaidya, Surgery, Ph.D. Surgery

3. Prof. Dr. Sarala Joshi, Ph.D. Nursing

Member

Member

4. Prof. Dr. Dhanik Lal Bharkher, MD, Ayurveda Member

5. Prof. Dr. Ram Kumar Ghimire, M.Sc. Radiology

Member

6. Assoc. Prof. Dr. Dinesh Kafle, MD, Orthopedics

Member

7. Mrs. Jamuna Sayami, Master in Nursing Member

8. Prof. Dr. Bharat Mani Pokhrel, Ph.D. Microbiology Member Secretary

9. Ms. Sharada Khanal, Asstt. Administrator Member Thus, IRB is responsible to guide, safety management, support, and creating positive environment as a facilitator rather than control to the researchers within the jurisdiction.

\section{References}

1. Cristina E.Torres, Ph.D. Social Science Professor and FERCAP Coordinator WHO-TDR Clinical Collaboration and Training Center: Paper presented at the Forum for Ethical Review Committees in Asia and the Western Pacific (FERCAP) July 2011, Bankok, Thailand

2. C.M. Gulhati, Monthly index of Medical Specialties

3. Gartoulla, RP (2008): Textbook on Medical Sociology/ Anthropology; RECID/Nepal

4. Gartoulla, RP (2011): Overview of Ethical Guidelines, Paper presented at the Consultative Meeting on Research Review Process organized by NHRC and Mary knoll Fathers and Brothers Project (9-10 September 2011).

5. NHRC (2011): National Ethical Guidelines For Health Research in Nepal and Standard Operating Procedures; NHRC, Kathmandu 